\title{
Acute kidney injury induces high-sensitivity troponin measurement changes after cardiac surgery
}

\author{
Amr S Omar 1,2,3*, Khaled Mahmoud 3,4,5, Samy Hanoura ${ }^{1,3,6}$, Hany Osman 1,6, Praveen Sivadasan ${ }^{1}$, Suraj Sudarsanan ${ }^{1}$, \\ Yasser Shouman ${ }^{1}$, Rajvir Singh ${ }^{7}$ and Abdulaziz AlKhulaifi ${ }^{1}$
}

\begin{abstract}
Background: The value of cardiac troponin as a risk assessment tool for cardiac disease in the setting of end-stage renal diseases (ESRD) is not equivalent to its value in those with normal renal function. This consideration had not been studied in settings of acute kidney injury (AKI). We aim to explore the diagnostic value of high sensitive troponin T (hsTnT) in the settings of cardiac surgery-induced AKl.

Methods: Single center observational retrospective study. Based on the AKI Network, patients divided into 2 groups, group I without AKI (259 patients) and group II with AKI (100 patients) where serial testing of hsTnT and creatine kinase (CK)-MB were followed in both groups. Patients with (ESRD) were excluded.

Results: The mean age in our study was $55.1 \pm 10.2$ years. High association of AKI (27.8\%) was found in our patients. Both groups were matched regarding the age, gender, body mass index, the association of diabetes or hypertension, and Euro score. AKI group had significantly higher mortality $5 \%$ vs group | 1.1\% ( $p=0.03)$. The hsTnt showed a significant sustained rise in the AKI group as compared to the non-AKI group, however CK-MB changes were significant initially but not sustained.

The AKI group was more associated with heart failure $17.9 \%$ vs $4.9 \%(p=0.001)$; and post-operative atrial fibrillation, $12.4 \%$ vs $2.9 \%$ ( $p=0.005)$. Lengths of ventilation, stays in ICU and in hospital were significantly higher in the AKI group.

Conclusions: Unlike the CK-MB profile, the hsTnT showed significant changes between both groups all over the course denoting possible delayed clearance in patients with AKI.
\end{abstract}

Keywords: Acute kidney injury, High sensitive troponin, Cardiac surgery

\section{Background}

The ultimate role of cardiac biomarkers in cardiac surgery remains unresolved, but such biomarkers are currently used in international guidelines to monitor and define myocardial infarction. The ideal analytical infrastructure and target proteins should be highly sensitive for early and late diagnosis, highly specific, easy to use, and affordable. These proteins should improve patient outcomes and impact therapeutic modalities [1]. Three subunits form the

\footnotetext{
* Correspondence: a_s_omar@yahoo.com

'Department of Cardiothoracic Surgery/Cardiac Anaesthesia and ICU, Heart Hospital, Hamad Medical Corporation, DohaPO 3050Qatar

${ }^{2}$ Department of Critical Care Medicine, Beni Suef University, Beni Suef, Egypt Full list of author information is available at the end of the article
}

backbone of the troponin complex. The $\mathrm{C}$, I and $\mathrm{T}$ subunits have been studied previously and are located on the myofibrillar thin (actin) filament of striated (skeletal and cardiac) muscle. Cardiac muscle expresses the isoforms troponin $\mathrm{T}$ and $\mathrm{I}$, and cardiac troponin $\mathrm{T}(\mathrm{cTnT})$ and $\mathrm{I}$ $(\mathrm{cTnI})$ are more specific than creatine kinase $(\mathrm{CK})$ values for myocardial injury. Thus, these proteins may be elevated when CK-MB is not [2].

The risk assessment of cardiac troponin and other cardiac biomarkers in end-stage renal disease and normal renal function is not equivalent. Clinical decision making based on cardiac biomarkers in patients with renal diseases requires justification in relation to patient management or outcomes [3]. 
A wide variety of causes may be associated with increases in cardiac enzymes after surgery, including acute coronary syndrome (ACS) related to recent acute myocardial infarction (AMI) before surgery [4] or perioperative myocardial infarction (PMI) directly related to cardiac surgery. These enzymes may also be elevated due to incomplete cardio-protection, reperfusion injury, and direct surgical trauma [5]. Elevation during cardiac surgery may not be ACS-related because such elevations may be caused by end-stage renal disease [6], acute pericarditis [7], acute heart failure (AHF), sepsis [8] and rhabdomyolysis [9].

PMI has a variable incidence and is reported after coronary artery bypass surgery (CABG) [10]. This phenomenon occurs both on and off pump, as well as in valvular surgeries, although it is relatively unusual. The reported incidence is up to $5 \%$ after CABG and $4 \%$ after valvular surgeries $[11,12]$.

The recently introduced high-sensitivity troponin $\mathrm{T}$ (hsTnT) showed higher sensitivity and negative predictive values than conventional cTn [13]. This marker exhibits excellent diagnostic performance, even with early presentation to the emergency department in the AMI scenario [14]. There is some evidence that hsTnT may possess better diagnostic accuracy than cTn [15]. hsTnT was introduced in 2010 and has been available at Hamad Hospital since June 2011. This protein not only serves as a sensitive and rapid diagnostic tool but may also aid in short- and long-term mortality prediction.

Cardiac troponin is related to short- and long-term mortality in the setting of cardiac surgery [16]. PMI is diagnosed based on a CK-MB increase of more than 10fold (99th percentile upper reference level) in combination with electrocardiogram (ECG), echocardiographic or angiographic evidence [17]. Acute kidney injury (AKI) is a frequent complication after cardiac surgery, with a reported incidence of up to $30 \%$. Renal insufficiency and acute cerebral events were associated with hsTnT elevation in the emergency department in a cross-sectional study performed by Lindner et al. [18].

No previous studies highlighted the influence of AKI on cardiac hsTnT. In a recent study performed by Prowle and Kirwan, the authors reported that long-term outcomes could be influenced after AKI in cardiac surgery, but cardiac troponins must be studied in these settings [19]. We hypothesize that hsTnT could be subjected to changes in the settings of AKI after cardiac surgery.

\section{Aim of the work}

Our goal was to explore the diagnostic value of hsTnT in the setting of cardiac surgery-induced AKI (with or without cardiac events).

\section{Methods}

1. Study design and assessments

This study was a single-center retrospective observational study that was conducted over 18 months (March 2011 to September 2013) in a 12-bed cardiothoracic ICU at Hamad Medical Corporation in Doha, Qatar. The ethical committee approved the study (reference number 15068/15), and written informed consent was waived for all subjects. We excluded patients with sepsis, preexisting high levels of hsTnT, end-stage renal disease (ESRD), and AKI before the surgery, as well as patients with marked intraoperative hypotension (mean arterial blood pressure less than 33\% of the initial value for more than $10 \mathrm{~min}$ ) [20]. A total of 19 patients were excluded. We recruited 359 consecutive patients who underwent cardiac surgery. The sample size was calculated to be a minimum of 320 subjects [21]. The following data were recorded for all patients: age, sex, existence of diabetes or hypertension, surgery type, length of anesthesia, cardiopulmonary bypass (CPB) time, aortic cross clamp (ACC) time, use of vasopressors and inotropes, and Euro SCORE. Outcome variables, including the length of mechanical ventilation (LOV), the length of stay in the ICU ( $\left.\mathrm{LOS}_{\mathrm{ICU}}\right)$ and the length of stay in the

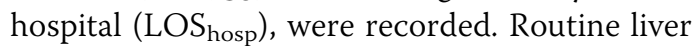
and renal functions were recorded. Complications, including AKI, post-operative atrial fibrillation (POAF), infection, stroke, and in-hospital mortality, were recorded for each patient. Dendrite Clinical Systems (London, UK) was used to retrieve data. Blood samples for hsTnT and CK-MB measurements were collected every six hours for the first $24 \mathrm{~h}$ after surgery and according to clinical indications after the first $24 \mathrm{~h}$. hsTnT was measured using COBAS Troponin T hs (highly sensitive) on a STAT (short turn-around time) instrument (Roche Diagnostics). ECGs were performed routinely before and immediately after surgery and every $12 \mathrm{~h}$ after surgery. Transthoracic echocardiography was performed for all patients before surgery and was then requested when indicated to trace new regional wall motion abnormalities in patients with high levels of cardiac enzymes and in patients requiring high doses of inotropes.

\section{Outcome definitions}

The primary outcome was association of hsTnT with post cardiac surgery AKI, the later was defined according to the consensus definition proposed by the Acute Kidney Injury Network. Here, AKI was defined as an abrupt (within $48 \mathrm{~h}$ ) reduction in 
kidney function, which was defined as an absolute increase in the serum creatinine concentration of $0.3 \mathrm{mg} / \mathrm{dL}$ or greater $(26.4 \mu \mathrm{mol} / \mathrm{L})$ or a percentage increase of $50 \%$ or greater (1.5-fold from baseline) [22]. The secondary outcome measures were the length of mechanical ventilation (LOV), the

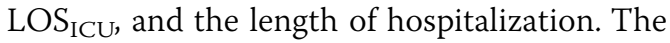
patients were divided into two groups based on AKI status: Group I experienced no AKI, and Group II experienced AKI.

3. Normally distributed continuous variables were expressed as the mean \pm standard deviation (SD). Skewed variables were presented as the median (interquartile range (IQR). The patients were divided into two groups based on the existence of AKI. Continuous variables were compared using the Student's $t$-test and the Mann Whitney $U$ test, as appropriate. Chi-square or Fisher's exact tests were used to compare categorical variables between the two groups. A significant association was defined by a probability $(\mathrm{P})$ value $\leq 0.05$ (two-tailed). HsTnT was converted to natural logarithm values for the purpose of multivariate linear regression analysis. Linear multivariate analysis putting the outcome variables was done in relation to HsTnT, Statistical analysis was performed using the SPSS software (version 22, Chicago, IL, USA).

\section{Results}

Of the 378 patients screened, 359 patients were enrolled in the study; the remaining 19 patients met one or more of the exclusion criteria. Males were predominant in the study population of 303 patients $(84.9 \%)$. The mean age was $57.2 \pm 11.6$ years, and the majority of patients underwent CABG surgery (291 patients (82.6\%); Tables 1 and 2).

Table 1 Description of the studied group

\begin{tabular}{llllc}
\hline Variable & Number & Minimum & Maximum & Mean \pm SD \\
\hline Age & 359 & 18 & 75 & $57.2 \pm 11.6$ \\
BMI $\left(\mathrm{kg} / \mathrm{m}^{2}\right)$ & 358 & 17.1 & 46.9 & $28.1 \pm 7.1$ \\
Creatinine (micromole/L) & 359 & 54.1 & 178.7 & $97.1 \pm 60.1$ \\
EF\% & 355 & 20 & 66 & $50.1 \pm 10.1$ \\
Additive Euro score & 354 & 0 & 18 & $3.6 \pm 2.9$ \\
CPB time (minutes) & 356 & 0 & 344 & $120.7 \pm 56.3$ \\
ACC time (minutes) & 356 & 0 & 180 & $86.1 \pm 40.1$ \\
Anesthesia time (minutes) & 355 & 200 & 648 & $301 \pm 81$ \\
LOV (minutes) & 354 & 210 & 4701 & $502 \pm 481$ \\
LOS & 354 & 6 & 499 & $35.1 \pm 30.6$ \\
\hline
\end{tabular}

$B M I$ body mass index, $E F$ ejection fraction, $C P B$ cardiopulmonary bypass, $A C C$ aortic cross clamp, WBCs white blood cells, LOV length of mechanical ventilation, $L O S_{\text {hosp }}$ Hospital length of stay
The patients were divided into two groups based on the existence of AKI. Group I consisted of patients with no AKI, and Group II consisted of patients with AKI (Tables 2 and 3). The two groups were well matched in terms of age, gender, hypertension, additive Euro score, body mass index (BMI), baseline creatinine and surgery type. A low ejection fraction (EF) was more notable in the AKI group (Table 2). The AKI group had significantly higher requirements for inotropes and vasopressors. There were no significant differences between the groups in terms of anesthesia time; however, $\mathrm{CPB}$ and ACC were significantly higher in the AKI group (Table 3).

The outcome variables, including the lengths of ventilation, ICU and hospital stay, were significantly higher in the AKI group. Post-operative complications, including post-operative atrial fibrillation (POAF), hospital-mortality and re-exploration, were significantly more frequent in the AKI group. No significant differences in the incidence of VAP and re-admission to the ICU were observed between groups. PMI was more frequent in patients with PMI (Table 3). Finally, Table 4 illustrates the relation of the outcome variables to HsTnT values.

Significant differences in hsTnT were observed between the two groups (Fig. 1) throughout the study period. The same changes were associated with CK-MB early in the study period but not later in the study period (Fig. 2).

\section{Discussion}

The salient findings of this study were as follows: 1) AKI patients exhibit changes in hsTnT but not CK-MB after cardiac surgery injury; 2) high mortality and morbidity were observed in the group with AKI in terms of

Table 2 Demographic differences between both groups

\begin{tabular}{|c|c|c|c|}
\hline Variable & $\begin{array}{l}\text { Group I (No AKI) } \\
259(\%)\end{array}$ & $\begin{array}{l}\text { Group II (AKI) } \\
100(\%)\end{array}$ & $P$-Value \\
\hline Age & $54.43 \pm 10.8$ & $56.09 \pm 10.7$ & 0.13 \\
\hline Gender (male) & $222(85.7)$ & $83(83)$ & 0.34 \\
\hline Diabetes & $138(53.2)$ & $56(56)$ & 0.38 \\
\hline Hypertension & $124(47.8)$ & $59(59)$ & 0.13 \\
\hline Euro score & $3.8 \pm 2.4$ & $5.1 \pm 3.6$ & 0.06 \\
\hline BMI & $28.1 \pm 5.8$ & $28.9 \pm 6.2$ & 0.12 \\
\hline$E F<40$ & $14(5.4)$ & $21(21)$ & 0.001 \\
\hline Elective surgery & $165(63.7)$ & $57(57)$ & 0.065 \\
\hline $\begin{array}{l}\text { Basal creatinine } \\
\text { (micromole/L) }\end{array}$ & $88.2 \pm 41.7$ & $92.1 \pm 42.3$ & 0.6 \\
\hline \multicolumn{4}{|l|}{ Surgery } \\
\hline CABG & $212(82.4)$ & $79(79)$ & 0.6 \\
\hline Valvular & $30(16)$ & $18(18)$ & 0.7 \\
\hline Aortic disssection & $4(1.3)$ & $1(1)$ & \\
\hline
\end{tabular}


Table 3 Main differences in both studied groups

\begin{tabular}{|c|c|c|c|}
\hline Variable & $\begin{array}{l}\text { Group I (No AKI) } \\
259(\%)\end{array}$ & $\begin{array}{l}\text { Group II (AKI) } \\
100(\%)\end{array}$ & P-Value \\
\hline \multicolumn{4}{|l|}{ Inotrops } \\
\hline Dopamine & $19(7.3)$ & $11(11)$ & 0.05 \\
\hline Adrenaline & $12(4.6)$ & $14(14)$ & 0.05 \\
\hline Noradrenline & $34(13.1)$ & $24(24)$ & 0.03 \\
\hline Dobutamine & $2(0.9)$ & $2(2)$ & 0.7 \\
\hline \multicolumn{4}{|l|}{ Intraoperative parameters } \\
\hline CPB time (minutes) & $119 \pm 43$ & $135 \pm 69.6$ & 0.06 \\
\hline ACC time (minutes) & $77.1 \pm 33$ & $77.4 \pm 48.5$ & 0.09 \\
\hline Anesthesia time (minutes) & $6.8 \pm 1.4$ & $6.7 \pm 2$ & 0.9 \\
\hline \multicolumn{4}{|l|}{ Postoperative parameters } \\
\hline LOV(minutes) & $364.1 \pm 112$ & $575.5 \pm 199$ & 0.001 \\
\hline 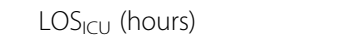 & $52.9 \pm 41.1$ & $109.4 \pm 89$ & 0.000 \\
\hline LOS $_{\text {hosp }}$ (days) & $10.1 \pm 3.4$ & $16.7 \pm 4.7$ & 0.001 \\
\hline \multicolumn{4}{|l|}{ Post-Operative outcome } \\
\hline POAF & $7(2.7)$ & $12(12)$ & 0.005 \\
\hline Inhospital-mortality & $3(1.1)$ & $5(5)$ & 0.03 \\
\hline VAP & $5(1.9)$ & $2(2)$ & 0.8 \\
\hline Re-admission ICU & $8(3)$ & $6(6)$ & 0.07 \\
\hline Re-exploration & $24(6.5)$ & $11(26.8)$ & 0.02 \\
\hline PMl & $2(0.8)$ & $6(6)$ & 0.02 \\
\hline
\end{tabular}

$A K I$ acute kidney injury, $C P B$ cardiopulmonary bypass, $A C C$ aortic cross clamp, LOV length of mechanical ventilation, LOS ICU ICU length of stay, LOS hosp Hospital length of stay, POAF post-operative atrial fibrillation, VAP ventilator associated pneumonia

POAF events and re-exploration; and 3) the AKI group exhibited prolonged lengths of ventilation, ICU and hospital stay. The utility of sensitive cardiac troponin for the early diagnosis of acute cardiac events has already been demonstrated [14]. The predictive value of cardiac troponin for patient outcomes in ESRD has been reported for a long time [5]. In addition, the incidence of AKI and the potential impact on patients undergoing cardiac surgery was elucidated in some previous studies [19]. Meanwhile, the impact of AKI on

Table 4 Outcome variables in relation to HsTnT

\begin{tabular}{llc}
\hline & Beta coefficient & Significance \\
\hline AKI & .47 & 0.001 \\
peri op MI & .91 & 0.013 \\
LOS HOSP (days) $_{\text {LOV (hours) }}$ & .005 & 0.090 \\
POAF & .004 & 0.001 \\
Mortality & .82 & 0.06 \\
CONSTANT & 1.1 & 0.2 \\
\hline AKI & 6.81 & 0.001
\end{tabular}

$A K I$ acute kidney injury, $P M I$ perioperative myocardial infarction, $L O S_{\text {hosp }}$ Hospital length of stay, LOV length of mechanical ventilation, POAF postoperative atrial fibrillation

Linear multivariate analysis for troponin values

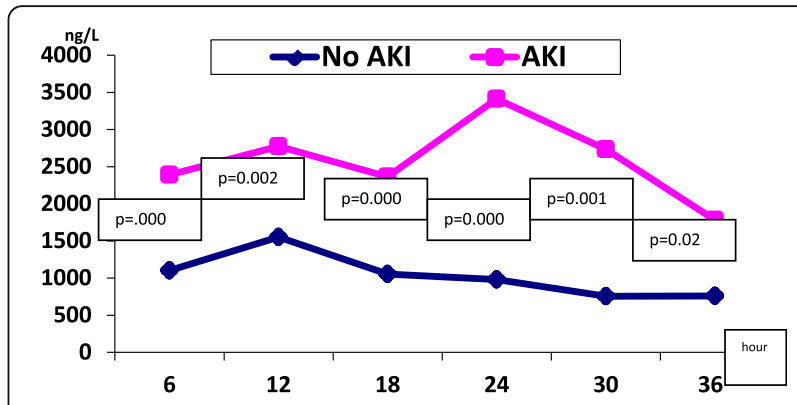

Fig. 1 Changes in hsTnT in both groups

cardiac troponins and their implications after cardiac surgery have not yet been studied.

To our knowledge, this study is the first to evaluate the diagnostic performance of hsTnT in the setting of cardiac surgery-induced AKI. This study showed that hsTnT may be an inaccurate marker of perioperative myocardial infarction in those patients who develop AKI post cardiac surgery. We showed that hsTnT is raised in AKI patients independent of cardiac events. While a few studies have addressed troponin in AKI [23], we are not aware of any studies that investigated hsTnT.

Rosner and Okusa reported previously that the pathogenesis of AKI associated with cardiac surgery involves multiple pathways. These pathways include inflammatory, hemodynamic, and nephrotoxic factors and may include other factors that are involved and overlap with each other, leading to kidney injury [24]. Risk factors for AKI have been identified in some clinical trials, and these factors can be used to determine the risk for AKI in patients who undergo cardiac surgery. The major risk factors include diabetes mellitus, hypertension, female gender, heart failure and a low EF [24].

In our study cohort, we encountered AKI in $27.8 \%$ of patients, which is within the range reported in the literature [24]. However, we believe that this value is relatively high. This high percentage could be attributed to the high incidence of diabetes mellitus, hypertension and

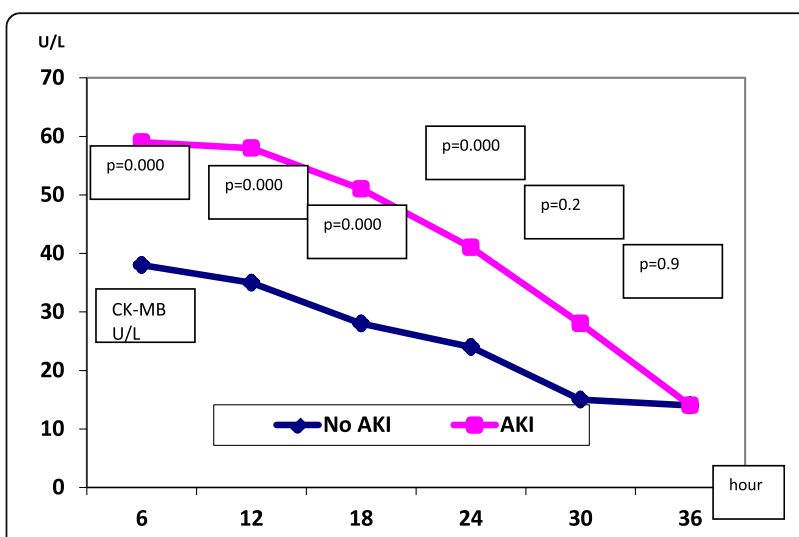

Fig. $\mathbf{2}$ Changes in CK-MB in both groups 
low EF in the study group. These factors would expose the patients to a higher risk of developing AKI.

Acute kidney injury is strongly associated with poor outcomes in terms of morbidity and mortality-especially in the setting of cardiac surgery [24, 25]. In our study, mortality and other outcome measures were significantly higher in the AKI group than the non-AKI group. Most outcome parameters were significantly higher in the AKI group. The requirement for more inotropic support, the length of ventilation (LOV), the $\mathrm{LOS}_{\mathrm{ICU}}$ and the $\mathrm{LOS}_{\text {Hosp }}$ were significantly elevated. These findings are consistent with other clinical reports in which high mortality and more complicated hospital courses were encountered in patients who underwent cardiac surgery and developed AKI.

Mao et al. performed a prospective, multicenter cohort study of 1,219 patients and confirmed the relationship with important clinical outcomes, including a longer length of hospital and ICU stay and a higher risk of dialysis or death [25]. However, the occurrence of AKI could be a sensitive indicator of the severity of the perioperative insult and poor physiological reserves, as well as a direct cause of the poor outcome [19]. Similarly we found changes in the outcome variables with higher levels of HsTnT (Table 4).

In patients with chronic kidney disease, it is possible to have elevated cardiac troponins even in the absence of true cardiac injury. This elevation could occur due to different processes, including decreased clearance and possible re-expression of cardiac troponin in uremic myopathic skeletal muscles [2]. Meanwhile, several studies have reported that increased cardiac troponin in endstage renal disease patients is an independent predictor of both short- and long-term mortality [5, 26].

In addition, elevated cardiac troponin levels in AKI patients post-cardiac surgery result in different effects, including myocardial cell injury due to incomplete cardioprotection, direct surgical trauma and possible reperfusion injury. This observation does not reflect perioperative myocardial infarction [2]. Here, we found that significant POAF events were associated with the AKI group. This association was also reported in a recent study by Kristovic et al. [27].

In our study cohort, we demonstrated a persistent rise in cardiac hsTnT in the AKI group across the entire time course. This pattern was not observed for the CK-MB marker. This trend of rising hsTnT in the setting of AKI should be considered when evaluating the diagnostic performance of hsTnT in patients with recent cardiac surgery for a cardiac insult-especially in the absence of a clear hsTnT diagnostic discrimination point. Omar et al. defined values $(3,466 \mathrm{ng} / \mathrm{L}$ and 2,309 $\mathrm{ng} / \mathrm{L})$ for hsTnT that accompany PMI and myocardial injury, respectively [28]. We found that PMI events were significantly more frequent in patients with AKI (Table 3), PMI was associated with high levels of HsTnT (Table 4). Perhaps when AKI develops after cardiac surgery, the biochemical diagnosis of a new cardiac insult using cardiac hsTnT should be based on a rising trend rather than a parallel rise or a defined value while keeping in mind the expected rise in hsTnT throughout the AKI course.

Study strengths, limitations and recommendations:

To our knowledge, this study is the first study to describe hsTnT changes in the setting of AKI. However, the findings should be interpreted with some caution. We recognize some limitations of our study, such as the short follow-up period, the retrospective design, and the lack of cut-off values for CK-MB and hsTnT, as well as the lack of a correlation with creatinine levels. The study was conducted in one center, and long-term follow up of the patients was impossible due to the highly dynamic work conditions in Qatar. These limitations could be further addressed in future studies.

\section{Conclusion}

Unlike the CK-MB assay, hsTnT exhibited significant changes in the AKI group, suggesting possible delayed clearance. A rise in hsTnT should be interpreted cautiously as a marker of post-operative myocardial injury and infarction in patients with AKI.

\section{Abbreviation}

ACC: Aortic cross clamp; AKI: Acute kidney injury; AMI: Acute myocardial infarction; CK-MB: Creatine kinase MB; CPP: Cardiopulmonary bypass time; EF: Ejection fraction; ESRD: End stage renal disease; hsTntT: High sensitive troponin T; ICU: Intenzsive care unit; LOS Hosp: Length of stay in hospital stay;

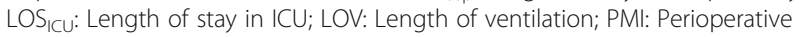
myocardial infarction; POAF: Post-operative atrial fibrillation

\section{Acknowledgment \\ This work would not have been possible without the kind support and help of many individuals and our organization. The authors thank all members of the Cardiothoracic surgery department, Heart Hospital, of Hamad Medical Corporation, Qatar, for providing necessary information regarding the project and for support. The authors also thank the members of the medical research department of Hamad Medical Corporation for their support throughout this project.}

\section{Funding}

The study was funded by Hamad Medical Corporation. The medical research center in Hamad Medical Corporation funded publishing this manuscript.

\section{Availability of data and materials}

The datasets supporting the conclusions of this article are available in the medical research center. The data is available by request to the corresponding author Dr. Amr Salah Omar by e-mail: a_s_omar@yahoo.com, after permission from the medical research center.

\section{Authors' contribution}

ASO: study design, contribution to concept, writing the manuscript and revising the final form. KM: writing and discussion management. $\mathrm{SH}$ : data collection and manuscript revision; HO: data collection, PS: data management and manuscript revision, data collection, interpretation of data, and revising of the manuscript, SS, data collection and data management; YS manuscript revision, initial design, RS: statistical analysis, AZK: suggested the idea, as a chair of the department provided general support and substantial contribution to concept and design, acquisition of data. All authors read and approved the final manuscript. 


\section{Competing interest}

The authors declare that they have no competing interests.

\section{Consent for publication}

Not applicable.

\section{Ethics approval and consent to participate}

This study was approved by medical research center in Hamad Medical Corporation. The ethical committee in Hamad medical corporation approved the study (reference number 15068/15), informed consent was waived by the ethical review panel for all patients enrolled in the study. However, all study data were maintained anonymously.

\section{Author details}

'Department of Cardiothoracic Surgery/Cardiac Anaesthesia and ICU, Heart Hospital, Hamad Medical Corporation, DohaPO 3050Qatar. ${ }^{2}$ Department of Critical Care Medicine, Beni Suef University, Beni Suef, Egypt. ${ }^{3}$ Weill Cornell Medical College-Qatar, Doha, Qatar. ${ }^{4}$ Department of Nephrology, Hamad Medical Corporation, DohaPO 3050Qatar. ${ }^{5}$ Department of Nephrology, Faculty of Medicine, Mansoura University, Mansoura, Egypt. ${ }^{6}$ Department of Anesthesia, Al-Azhar University, Cairo, Egypt. ${ }^{7}$ Department of Cardiology Research Center, Hamad Medical Corporation, Doha, Qatar.

Received: 3 April 2016 Accepted: 19 January 2017

\section{Published online: 31 January 2017}

\section{References}

1. Dolci A, Panteghini M. The exciting story of cardiac biomarkers: From retrospective detection to gold diagnostic standard for acute myocardial infarction and more. Review. Clinica Chimica Acta. 2006;369:179-87.

2. Korff S, Katus HA, Giannitsis E. Differential diagnosis of elevated troponins. Heart. 2006;92:987-93.

3. Vesely DL. Natriuretic peptides and acute renal failure. Am J Physiol Renal Physiol. 2003;285(2):F167-77.

4. Alpert JS, Thygesen K, Antman E, Ohman M, Sendon JLL, Rydén L, Tendera M. Myocardial infarction redefined - a consensus document of the Joint European Society of Cardiology/American College of Cardiology committee for the redefinition of myocardial infarction. J Am Coll Cardiol. 2000;36:959-69.

5. Apple FS, Murakami MM, Pearce LA, Herzog CA. Predictive value of cardiac troponin I and $T$ for subsequent death in end-stage renal disease. Circulation. 2002;106:2941-5.

6. Imazio M, Demichelis B, Cecchi E, Belli R, Ghisio A, Bobbio M, Trinchero R Cardiac troponin I in acute pericarditis. J Am Coll Cardiol. 2003;42(12):2144-8.

7. Perna ER, Macín SM, Parras Jl, Pantich R, Farías EF, Badaracco JR, Jantus E, Medina F, Brizuela M. Cardiac troponin T levels are associated with poor short- and long-term prognosis in patients with acute cardiogenic pulmonary edema. Am Heart J. 2002;143(5):814-20.

8. Ammann P, Fehr T, Minder El, Günter C, Bertel O. Elevation of troponin I in sepsis and septic shock. Intensive Care Med. 2001;27(6):965-9.

9. Lavoinne A, Hue G. Serum cardiac troponins I and T in early posttraumatic rhabdomyolysis. Clin Chem. 1998;44(3):667-8.

10. Alexiou K, Wilbring M, Koch C, Kappert U, Tugtekin S, Matschke K. Midterm follow-up of patients with perioperative myocardial infarction after coronary artery bypass surgery: clinical significance of different treatment strategies. Thorac Cardiovasc Surg. 2012;60(7):452-61.

11. Okamura Y, Takeuchi Y, Gomi A, Nagashima M, Mori H, Hattori J. Clinical evaluation of perioperative myocardial infarction as a complication of valave replacement. Kyobu Geka. 1989;42(12):1012-5. Japanes.

12. McGregor CG, MacLeod MD, Muir AL, Smith AF, Hannan WJ, Miller HC. Myocardial infarction related to valve replacement surgery. Br Heart J. 1984;51(6):612-7.

13. Freund $Y$, Gobeaux CC, Bonnet P, Claessens YE, Allo JC, Doumenc B, Leumani F, Cosson C, Riou B, Ray P. High-sensitivity versus conventional troponin in the emergency department for the diagnosis of acute myocardial infarction. Critical Care. 2011;15:R147.

14. Reichlin T, Hochholzer W, Bassetti S, Steuer S, Stelzig C, Hartwiger S, Biedert S, Schaub N, Buerge C, Potocki M, Noveanu M, Breidthardt T, Twerenbold R, Winkler K, Bingisser R, Mueller C. Early diagnosis of myocardial infarction with sensitive cardiac troponin assays. N Engl J Med. 2009;361:858-67.

15. Januzzi JL, Jr Bamberg F, Lee H, Truong QA, Nichols JH, Karakas M, Mohammed AA, Schlett CL, Nagurney JT, Hoffmann U, Koenig W. High- sensitivity troponin T concentrations in acute chest pain patients evaluated with cardiac computed tomography. Circulation. 2010;121:1227-34.

16. Lurati Buse GA, Koller MT, Grapow M, Bolliger D, Seeberger M, Filipovic M. The prognostic value of troponin release after adult cardiac surgery - a meta-analysis. Eur J Cardiothorac Surg. 2010;37(2):399-406.

17. Thygesen K, Alpert JS, Jaffe AS, Simoons ML, Chaitman BR, White HD. Joint ESC/ACCF/AHA/WHF Task Force for the Universal Definition of Myocardial Third universal definition of myocardial infarction. Eur Heart J. 2012;33(20):2551. 67.

18. Lindner $G$, Pfortmueller CA, Braun $C T$, Exadaktylos AK. Non-acute myocardial infarction-related causes of elevated high-sensitive troponin $\mathrm{T}$ in the emergency room: a cross-sectional analysis. Intern Emerg Med. 2014;9(3):335-9.

19. Prowle JR, Kirwan CJ. Acute Kidney Injury After Cardiac Surgery: The Injury That Keeps on Hurting?*. Crit Care Med. 2014;42(9):2142-3.

20. Ahuja K, Charap MH. Management of perioperative hypertensive urgencies with parenteral medications. J Hosp Med. 2010;5(2):E11-6.

21. Charan J, Biswas T. How to calculate sample size for different study designs in medical research? Indian J Psychol Med. 2013;35(2):121.

22. Mehta RL, Kellum JA, Shah SV, Molitoris BA, Ronco C, Warnock DG, Levin A. Acute Kidney Injury Network (AKIN): report of an initiative to improve outcomes in acute kidney injury. Crit Care. 2007;11(2):R31.

23. Song D, de Zoysa JR, Ng A, Chiu W. Troponins in acute kidney injury. Ren Fail. 2012;34(1):35-9.

24. Rosner MH, Okusa MD. Acute kidney injury associated with cardiac surgery. Clin J Am Soc Nephrol. 2006;1(1):19-32.

25. Mao H, Katz N, Ariyanon W, Blanca-Martos L, Adýbelli Z, Giuliani A, Ronco C. Cardiac surgery-associated acute kidney injury. Cardiorenal Med. 2013;3(3):178-99.

26. Dierkes J, Domrose U, Westphal S, Ambrosch A, Bosselmann P, Neumann $\mathrm{KH}$, Luley C. Cardiac troponin T predicts mortality in patients with end-stage renal disease. Circulation. 2000;102:1964-9.

27. Kristovic D, Horvatic I, Husedzinovic I, Sutlic Z, Rudez I, Baric D \& Crnogorac M. Cardiac surgery-associated acute kidney injury: risk factors analysis and comparison of prediction models. Interact Cardiovasc Thorac Surg. 2015;21(3): 366-73.

28. Omar AS, Sudarsanan S, Hanoura S, Osman H, Sivadasan PC, Shouman Y, Al Khulaifi A. Kinetics of Highly Sensitive Troponin T after Cardiac Surgery. BioMed Res Intern. 2015;2015:574546.

\section{Submit your next manuscript to BioMed Central and we will help you at every step:}

- We accept pre-submission inquiries

- Our selector tool helps you to find the most relevant journal

- We provide round the clock customer support

- Convenient online submission

- Thorough peer review

- Inclusion in PubMed and all major indexing services

- Maximum visibility for your research

Submit your manuscript at www.biomedcentral.com/submit
) Biomed Central 